\title{
CAPA FÍSICA Y ALGORITMOS DE PLANIFICACIÓN DE ENLACE DESCENDENTE EN LTE Y WiMAX
}

\section{PHYSICAL LAYER AND DOWNLINK SCHEDULING ALGORITHMS IN LTE AND WiMAX}

\section{MSc. Ernesto Cadena Muñoz", MSc. PhD (c) Hermes Javier Eslava Blanco."* PhD. Ingrid Patricia Páez Parra.*}

\author{
* Servicio Nacional de Aprendizaje-SENA - Universidad Nacional de Colombia, \\ Centro de Electricidad, Electrónica y Telecomunicaciones, Grupo de Investigación GICS. \\ Carrera 30, No. 17B-25 Sur, Tel. (+571) 5960074. \\ E-mail: ecadenam@misena.edu.co, ecadenam@unal.edu.co. \\ ** Universidad Distrital Francisco José de Caldas - UNAL, Facultad Tecnológica, \\ Ingeniería de Telecomunicaciones, Grupo de Investigación TELETECNO. \\ Carrera 7, No. 40B - 53, Bogotá D.C, Colombia, Tel. (+571) 3239300 Ext 5415. \\ E-mail: hjeslavab@udistrital.edu.co, heslavab@unal.edu.co \\ **** Universidad Nacional de Colombia, Facultad de Ingeniería, Ingeniería Sistemas e \\ Industrial, Grupo de Investigación GITUN. \\ Carrera 30, No. 45, Bogotá D.C, Colombia, Te. (+571) 3165000 Ext. 14089. \\ E-mail: ippaezp@unal.edu.co.
}

Resumen: Este trabajo presenta una revisión de la capa física y los algoritmos de planificación en LTE y WiMAX, como son BestCQI, PF y RR en la asignación de recursos para el enlace descendente o Downlink. Se presentan los principales criterios a tener en cuenta en los planificadores como la fairness y el rendimiento, a partir del análisis de las tramas, subtramas, señalización y sincronización que componen la capa física de LTE y WiMAX. Finalmente se presentan las conclusiones de los tres algoritmos de planificación de recursos y se muestran las debilidades y fortalezas de cada uno.

Palabras clave: Fairness, LTE, resource allocation, scheduling algorithm, throughput.

\begin{abstract}
This paper presents an overview of the physical layer and the scheduling algorithms in LTE and WiMAX such a Best CQI, PF and RR for resource allocation in Downlink Channel. The main criteria to consider in scheduling are fairness and performance, based on the analysis of the frame, sub-frames, signaling and synchronism that is done for physical layer. Finally the conclusions of the three algorithms and the strength and weaknesses of each one are presented.
\end{abstract}

Keywords: Fairness; LTE, resource allocation, scheduling algorithm, throughput.

\section{INTRODUCCIÓN}

Los servicios de telecomunicaciones que se prestan hoy en día son el resultado de una continua evolución de la microelectrónica y el desarrollo de software especialmente durante la última década. Debido a esta evolución, los sistemas de comunicaciones móviles deben satisfacer la demanda creciente de velocidad de conexión, calidad de servicio, video, televisión y soportar la movilidad del usuario, para lo cual reconocemos sistemas de telecomunicaciones móviles de diferentes generaciones siendo la actual la cuarta generación (4G) (Akan \& Edemen, 2010). 
LTE (Long Term Evolution) es un estándar desarrollado por el grupo 3GPP (3rd Generation Partnership Project), esta tecnología surge para dar soporte a aplicaciones móviles con requerimientos de ancho de banda y tasas de transmisión cada vez más exigentes. LTE esta diseñado para soportar multiplexación en tiempo, frecuencia y diferentes configuraciones del sistema de antenas (Dahlman, Parkvall, \& Skold, 2013).

LTE es una tecnología que de acuerdo con la UIT (Unión Internacional de Telecomunicaciones) fue considerada como $3.9 \mathrm{G}$, ya que no cumplía con los requisitos del IMT-Advanced (International Mobile Telecommunications-Advanced), en términos de velocidades de transmisión que debían ser iguales o superiores a 1 Gbps. Lo mismo ocurría para WiMAX (Worldwide Interoperability for Microwave Access) móvil 1.0 (IEEE 802.16e), lo que no permitía a los comercializadores de equipos ofrecer LTE y WiMAX como tecnologías de 4G. En diciembre de 2010, la UIT reconsidero su definición para sistemas $4 \mathrm{G}$ y dio su autorización para describir tecnologías LTE y WiMAX móvil 1.0 como sistemas 4G (Cox, 2012).

Dentro de los sistemas de 4G como LTE, LTEAdvanced y WiMAX la asignación de recursos de radio es un tema de gran importancia debido a que estos son escasos, costosos y sus condiciones varían en el tiempo, por lo tanto su administración debe hacerse de forma dinámica. El estándar de LTE define los conceptos generales de los denominados RRM (Radio Resource Management), pero no especifica los algoritmos a utilizar dejando su implementación y diseño a los fabricantes de equipos (Agusti et al., 2010). Actualmente hay una necesidad en las redes de $4 \mathrm{G}$ de administrar de forma más eficiente los recursos de nivel físico; La implementación de estos modelos es pertinente ya que la tecnología está siendo implementada hoy en día en nuestro país y proyecta ser el estándar utilizado para servicios de 4G.

Se debe estudiar la posibilidad de mejora de las arquitecturas tradicionales basadas en capas como el modelo OSI, que permitan la comunicación entre capas para optimizar el sistema y la comunicación general. Este tema sumado a la complejidad del modelamiento de 4G-LTE no ha sido abordado con suficiente profundidad y aún faltan estudios para complementar la posibilidad de ir estandarizando los diseños que se puedan realizar para esta tecnología buscando mejorar el manejo de la energía (Melodia, Vuran, \& Pompili, 2006), la asignación de recursos (Fang \& McDonald, 2004),
(Van Hoesel, Nieberg, Wu, \& Havinga, 2004), (Vuran, Gungor, \& Akan, 2005) o la optimización general (Ukil, 2009).

En la segunda sección se caracterizan los componentes y la arquitectura de la capa física de WiMAX incluyendo la composición de las tramas, subtramas, las señales de sincronización y señalización. En la tercera sección se realiza el mismo análisis con LTE. En la cuarta sección se realiza una descripción de tres técnicas utilizadas para la planificación de recursos en enlaces de bajada de LTE y finalmente en la quinta sección se presentan las conclusiones.

\section{CAPA FÍSICA WiMAX}

El estándar 802.16 soporta varios tipos de modulaciones en la capa física, la primera de ellas SC (Single Carrier) está diseñada para frecuencias entre 10 a $60 \mathrm{GHz}$ y requiere LOS (Line Of Sight). Por esta razón, no se encuentran muchos equipos comerciales, para la versión de WiMAX fijo (802.16-2004) fijo con frecuencias entre $2-11 \mathrm{GHz}$ se emplea OFDM (Orthogonal Frequency Division Multiplexing), en este caso no se requiere línea de vista, alcanza velocidades de hasta 75 Mbps y alcance hasta de $10 \mathrm{~km}$. Para la versión de WiMAX móvil (802.16e) se emplea OFDMA (Orthogonal Frequency Division Multiple Access), que opera en un rango de frecuencias entre $2-6 \mathrm{GHz}$, alcanzando velocidades de transmisión de hasta $30 \mathrm{Mbps}$ y un alcance máximo de $3.5 \mathrm{Kms}$ (Chakchai So-In, Jain, \& Tamimi, 2009) (Castellanos \& Khan, 2010a).

Como puede apreciarse en la Fig. 1, la asignación de recursos cuando se emplea el SC, es más simple ya que sólo considera el dominio del tiempo, los usuarios utilizan la misma portadora y comparten en canal a intervalos regulares de tiempo. En OFDM se cuenta con subcanales conformados por subportadoras a diferentes frecuencias e intervalos regulares de tiempo, la asignación en OFDMA debe considerar tanto el tiempo como la frecuencia aumentando la complejidad del scheduler.
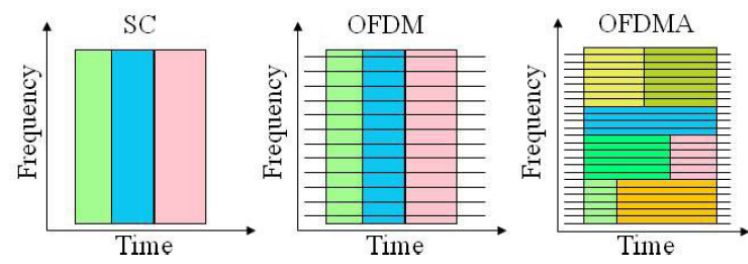

Fig. 1. Capa física WiMAX.

(Chakchai So-In et al., 2009). 


\subsection{Subcanalización en WiMAX}

No todas las portadoras en OFDM son utilizadas para transmitir datos, existen 4 tipos de subportadoras. Subportadoras de datos: utilizadas para transmitir datos, Subportadoras pilotos: son utilizadas con fines de sincronización, Subportadoras Nulas: empleadas como guardas, Subportadoras DC: correspondiente a la frecuencia central RF. El número de subportadoras está determinado por el FFT utilizadas como puede apreciarse en la Fig. 2 (Nuaymi, 2007).

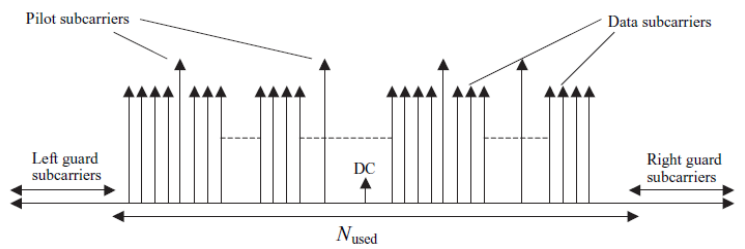

Fig. 2. Tipos de subportadoras OFDM. (Nuaymi, 2007).

\subsection{Estructura de trama WiMAX}

La trama WiMAX está conformada por dos subtramas: DL (Downlink) y UL (Uplink) separadas por guardas TTG (Transmit-receive Transition Gap), se presenta cuando la BS (Base Station) cambia del modo de transmisión a recepción y RTG (Receive-transmit Transition Gap) ocurre cuando la BS cambia de modo de recepción a transmisión. Como puede observarse en la Fig. 3 se tiene un preámbulo con fines de sincronización, seguido de un DL-MAP (Downlink Map) y UL-MAP (Uplink map) en donde se define cuando inicia y finaliza cada ráfaga de datos, el tipo de modulación a utilizar y el FEC (forward error control). El FCH (Frame Control Header) contiene información del tamaño de los mapas y las subportadoras utilizables.

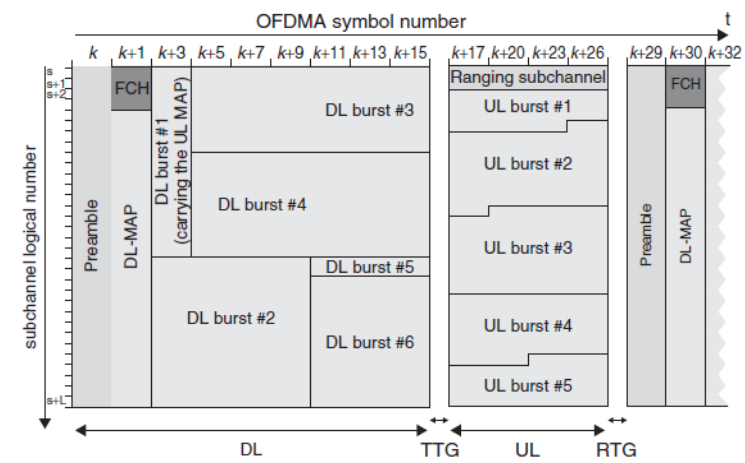

Fig. 3. Estructura de trama WiMAX.

(Taha, Ali, \& Hassanein, 2011).
Las condiciones del canal pueden cambiar y afectar la transmisión, WiMAX cuenta con modulación adaptativa que se puede ajustar de acuerdo con las condiciones del canal, mediante el CQI (Channel Quality Indicator), que monitorea y transmite la información de condición del canal para que la BS realice los ajustes (Chakchai So-In et al., 2009) (Nuaymi, 2007) (Taha et al., 2011) .

\subsection{Calidad de servicio en redes WiMAX}

A diferencias de las redes wifi en donde todas las aplicaciones reciben el mimo nivel de QoS, WiMAX ofrece cinco clases QoS según el tipo de aplicación que se esté utilizando, es así como en el control de admisión limita el número de conexiones a la red, examinando el tipo de QoS que demanda cada SF, estableciendo si estos requerimientos pueden ser atendidos. La información del usuario proveniente de capas superiores viene marcada con un TOS (Type of Service ) sobre cada paquete, en la capa MAC el paquete es clasificado según la clase de servicio. Las cinco clases de servicio soportadas por WiMAX son: (Castellanos \& Khan, 2010b).

UGS (Unsolited Grant Services): está diseñado para soportar flujos de datos en tiempo real, que se caracteriza por que sus paquetes de datos tienen un tamaño fijo, con un CBR, se emiten a intervalos periódicos. Como ejemplo se tienen los sistemas T1/E1, en la transmisión de señales telefónicas basadas en PCM y voz sobre IP con supresión de silencios, busca mantener una baja latencia y bajo Jitter. En la clase de servicio UGS la BS provee recursos para la transmisión de datos de tamaño fijo a intervalos periódicos, eliminando de esta forma el overhead y la latencia en las SS cuando hacen requerimientos.

rtPS (Real-time Polling Services): Diseñado para soportar flujos de datos de tamaño variable que son emitidos a intervalos periódicos, esto podría ser el caso por ejemplo de video MPEG (Moving pintures experts Group). En esta clase de servicio las BS proveen oportunidades de solicitudes periódicas unicast en el UL con la cual establece las necesidades de los flujos de datos de tiempo real, permitiendo a las SS especificar el tamaño requerido. Este servicio requiere mayores sobre encabezados (overhead) que UGS pero soportar asignación de tamaño variable para optimizar el transporte eficiente de aplicaciones de tiempo real.

ertPS (Extended Real-Time Polling Services): Fue adicionado al estandar 802.16e, el ertPS es un 
algoritmo de planificación diseñado sobre las características de eficiencia de UGS y rtPS. En este caso la BS provee un mecanismo de concesión de recursos en forma periódica no solicitada al igual que en UGS, guardando los requerimientos de ancho de banda y latencia.

Sin embargo mientras las asignaciones UGS son de tamaño fijo en ertPS la asignación es dinámica. ertPS es una clase de servicio adecuado para aplicaciones de tiempo real de tasa variable con requerimientos de data rate y retardo por ejemplo voz sobre IP sin supresión de silencios.

nrtPS (Non Real-time Polling Services): está diseñado para soportar flujos de datos con tolerancia al retardo que consisten en transmisión de paquetes de datos de tamaño variable en los que se requiere un mínimo de data rate, el estándar considera que este puede ser el caso de transmisiones FTP.

BE (Best Effort): La clase de servicio BE está diseñado para soportar flujos de datos en los cuales no se garantiza un mínimo de calidad de servicio. Puede pasar periodos largos de tiempo sin que se transmita ningún paquete.

\subsection{Planificación de recursos en redes WiMAX}

El control de admisión de la BS (Base Station) limita el número de conexiones a la red examinando en cada SF (Service Flow) los requerimientos de QoS y verificando que estos puedan ser cumplidos de acuerdo con los recursos disponibles. La BS WiMAX cuenta con dos planificadores una para DL-MAP y otro para UL-MAP en donde la BS determina el orden en el cual serán atendidos los paquetes una vez son clasificados en una clase de servicio ya sea UGS, rtPS, ertPS, nrtPS, BE.

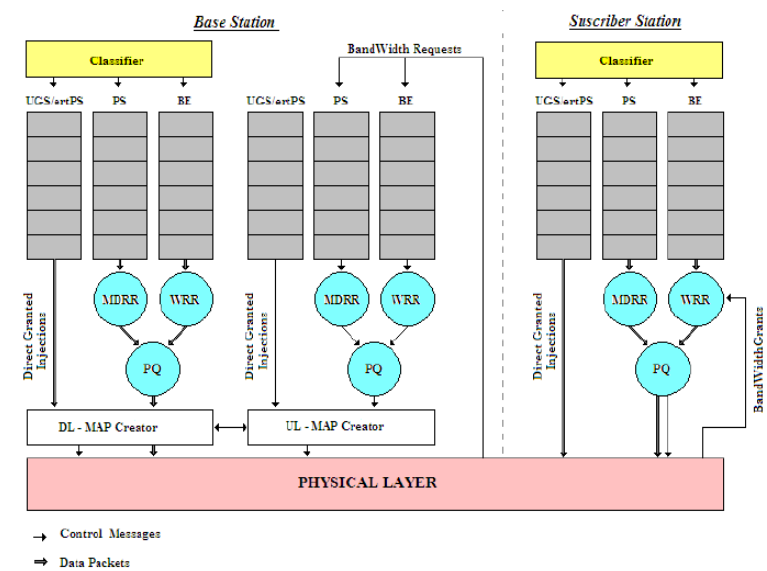

Fig. 4. Planificador WiMAX (G. Israel, 2013).
Como puede observarse en la Fig. 4 después de que el paquete es clasificado en una clase de servicio, el paquete pasa a una cola en donde debe esperar para ser transmitido. Las clases de servicio UGS/ertPS son atendidos directamente sin necesidad de someterse a ningún algoritmo de planificación, ya que sus paquetes son sensibles al retardo. Los servicios basados en PS como son rtPS, nrtPS son encolados y atendidos conforme al algoritmo de planificación MDRR (Modified Deficit Round Robin), los paquetes clasificados como BE son atendidos mediante el algoritmo WRR (Weighted Round Robin). Los paquetes PS tendrán prioridad sobre los clasificados como BE de tal forma que solo serán transmitidos si la cola PS se encuentra vacía (G. Israel, 2013).

\section{CAPA FÍSICA LTE}

La interfaz de radio del Sistema LTE se basa en la utilización de técnicas de modulación mutiportadora OFDMA en el DL, desde el eNodeB al UE (User Equiment) (Furht \& Ahson, 2009) y SCFDMA (Single Carrier - Frequency Division Multiple Access) en el UL.

El acceso múltiple al canal de radio es uno de los procedimientos más complejos en los sistemas de telecomunicaciones fijos y móviles. Se han desarrollado técnicas par que varios usuarios compartan un mismo canal; entre estas técnicas están: FDMA (Frequency Division Multiple Access), TDMA (Time Division Multiple Access), CDMA (Code division multiple Access) y SDMA (Space-division multiple Access).

OFMDA se basa en dividir el ancho de banda en un número elevado de subportadoras que cumplen con determinadas propiedades de ortogonalidad entre ellas. Cada subportadora transporta un símbolo de datos distinto, lo que permite adaptar la transmisión de cada símbolo a las condiciones de canal y combatir mejor los efectos de la propagación multitrayectoria. Cada subportadora es modulada utilizando diferentes niveles de modulación como QPSK (Quadrature Phase Shift Keying), QAM (Quadrature Amplitude Modulation), 64QAM (Dikamba, 2011). El problema es la separación de las señales $y$ es donde se necesitan técnicas avanzadas en función del método de acceso múltiple usado y del desempeño que se espera del sistema (G. Israel, 2013).

La ortogonalidad evita la aparición de interferencia entre subportadoras adyacentes y elimina la 
necesidad de bandas de guarda, mejorando la eficiencia espectral frente a soluciones tipo FDM. Se crea una estructura de recursos en tiempo y frecuencia que el nivel de control de acceso al medio MAC (Medium Access Control) deberá repartir entre los usuarios de la forma más eficiente posible como se puede ver en la Fig. 5. WiMAX y LTE soportan FDD (Frequency Division Duplex) y temporal TDD (Time Division Duplex), aunque LTE está más orientado a FDD y WiMAX a TDD. Se necesita una sincronización muy precisa y coordinación entre celdas para evitar la colisión entre tramas DL de una celda y UL de otra (G. Israel, 2013).

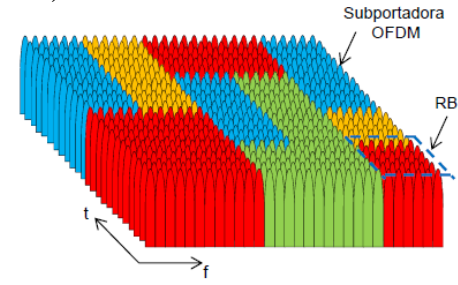

Fig. 5. Ejemplo de asignación espacio- temporal en OFDMA (G. Israel, 2013).

\subsection{Estructura de la trama LTE}

La organización de los recursos es una función muy importante tanto en frecuencia como en el tiempo y se organiza usando una rejilla de recursos, como se observa en la Fig. 6 para un prefijo cíclico normal. La unidad básica es el RE (resource element), se asigna un símbolo para cada subportadora. Los RE son agrupados en RBs (Resource Blocks), cada uno con $0.5 \mathrm{~ms}$ y 12 subportadoras. El eNodeB utiliza los RB para una planificación dependiente de la frecuencia, asignando símbolos y subportadoras con cada subtrama en RB (Cox, 2012).

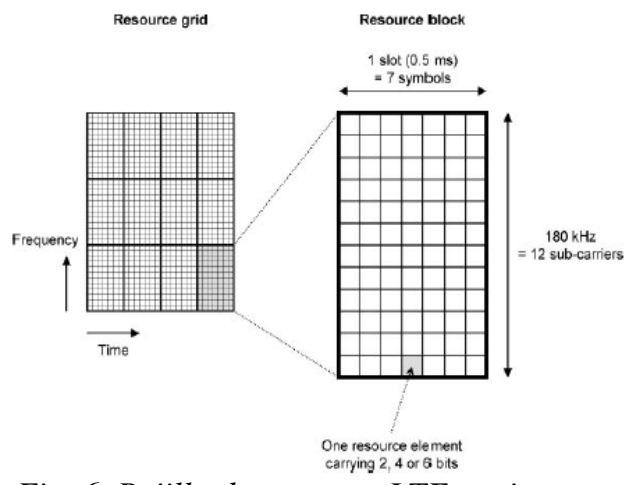

Fig. 6. Rejilla de recursos LTE en tiempo y frecuencia para un prefijo cíclico normal

(Cox, 2012)

Cada RE usualmente tiene 2, 4 o 6 bits de canal físico, dependiendo si el esquema de modulación es QPSK, 16-QAM o 64-QAM se muestra en Fig. 7.

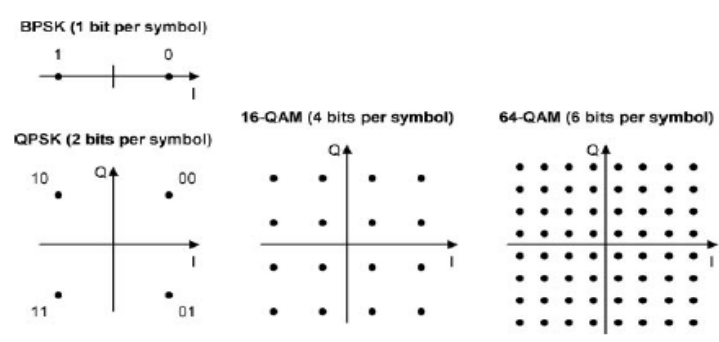

Fig. 7. Esquemas de Modulación en LTE

(Cox, 2012).

En la Fig. 8 se puede observar una subtrama, conformada por dos slot, cada uno con una duración de $0.5 \mathrm{~ms}$ y 12 subportadoras, con un $\Delta f$ de $15 \mathrm{KHz}$, para un total de $180 \mathrm{KHz}$. Cada subportadora contiene 6 o 7 símbolos OFDM, 7 para un prefijo cíclico normal. En cada TTI (Transmission Time interval) se tienen dos slots o dos RB que corresponden a un subtrama LTE (AlQahtani \& Alhassany, 2013).

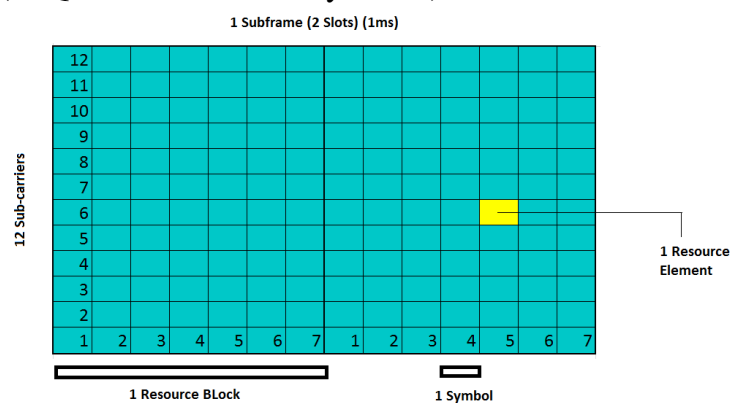

Fig. 8. Bloque de recursos físicos $R B$ (Agusti et al., 2010).

En DL las tramas están formada por 10 subtramas de $1 \mathrm{~ms}$ de duración cada una. Hay diez tramas en total para el UL y diez para el DL. Cada slot contiene 6 o 7 símbolos OFDM (Dikamba, 2011). Las estructuras de trama son de dos tipos:

\subsection{Estructura de trama tipo 1}

Es válida para sistemas que utilizan FDD y aplica tanto en DL como al UL. Soporta FDD tanto half como full duplex. El eje temporal se divide en tramas de $10 \mathrm{~ms}$. Cada trama contiene por 20 slots de duración 0,5 ms. Se define una unidad básica de recursos, formada por dos slots denominada subtrama. Esta estructura se muestra en la Fig. 9.

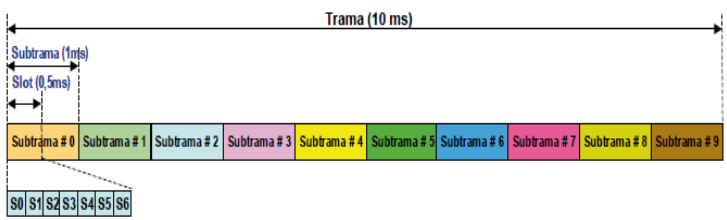

Fig. 9. Estructura de trama LTE Tipo 1 (Dikamba, 2011). 


\subsubsection{Estructura de trama tipo 2}

Diseñada para el modo TDD. Se divide en tramas de $10 \mathrm{~ms}$, cada una compuesta por 10 subtramas. Es más flexible que la tipo 1 , contiene subtramas de transmisión para el DL, el UL y subtramas especiales que contienen los símbolos piloto de los dos tipos, junto con periodos de guarda entre transmisión y recepción. Facilita la transición entre DL y UL por razones de sincronización. Aunque el sistema esté sincronizado, en UL las transmisiones siempre pueden sufrir una pequeña incertidumbre temporal que obliga a mantener un mínimo tiempo de guarda (Agusti et al., 2010). Esta trama se puede ver en la Fig. 10.

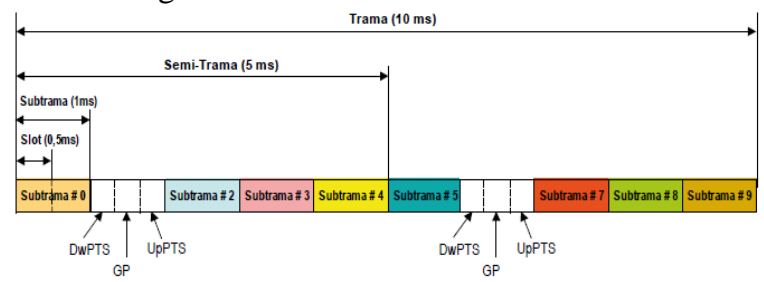

Fig. 10. Estructura de trama LTE Tipo 2. (3GPP TS 36.211, 2009).

El número de bloques disponibles depende directamente del ancho de banda; en modo FDD el número de RB permitidos de acuerdo al ancho de banda se resumen en la Tabla 1, (3GPP TS 36.101, 2009),(Motorola, White Paper),(Ikuno, Wrulich, \& Rupp, 2010). La cantidad de Subportadoras depende de la cantidad de bloques de recursos, que a su vez depende del ancho de banda del sistema. En la Tabla 1, se puede apreciar la cantidad de RB's , las subportadoras ocupadas y el tamaño de la FFT utilizada de acuerdo al ancho de banda.

\section{Tabla 1: Número de RB en función del ancho de banda (Dikamba, 2011).}

\begin{tabular}{|c|c|c|c|c|c|c|}
\hline $\begin{array}{c}\text { Ancho de } \\
\text { Banda }\end{array}$ & $1.4 \mathrm{MHz}$ & $3 \mathrm{MHz}$ & $5 \mathrm{MHz}$ & $10 \mathrm{MHz}$ & $15 \mathrm{MHz}$ & $20 \mathrm{MHz}$ \\
\hline RB & 6 & 15 & 25 & 50 & 75 & 100 \\
\hline $\begin{array}{c}\text { Número de } \\
\text { Subportadoras } \\
\text { Ocupadas }\end{array}$ & 72 & 180 & 300 & 600 & 900 & 1200 \\
\hline \begin{tabular}{c} 
Tamaño FFT \\
\hline
\end{tabular} & 128 & 256 & 512 & 1024 & 1536 & 2048 \\
\hline
\end{tabular}

\subsection{Señales Físicas en DL}

Para la implementación de los mecanismos físicos necesarios para la demodulación/detección de las señales moduladas OFDMA del enlace DL del sistema LTE, se han establecido un conjunto de señales físicas de referencia y de sincronización temporal, que se mencionan a continuación (Agusti et al., 2010):

\section{Señales de Referencia (RS)}

Las señales de referencia se utilizan para:

- Obtener medidas de calidad en el enlace descendente.

- Estimar la respuesta impulsional del canal para demodulación/detección coherente.

- Implementar mecanismos de búsqueda de celda y sincronización inicial.

Se sitúan en una subportadora y un símbolo, mantienen una separación en frecuencia de seis subportadoras. Se necesitan 2 señales de referencia por PRB denominadas RSP (Reference Signal Primary), ubicadas en el primer símbolo de un slot. $\mathrm{Si}$ se requiere, puede ubicarse un segundo grupo de señales piloto o RSS (Reference Signal Secundary), situadas en el quinto símbolo de una ranura temporal.

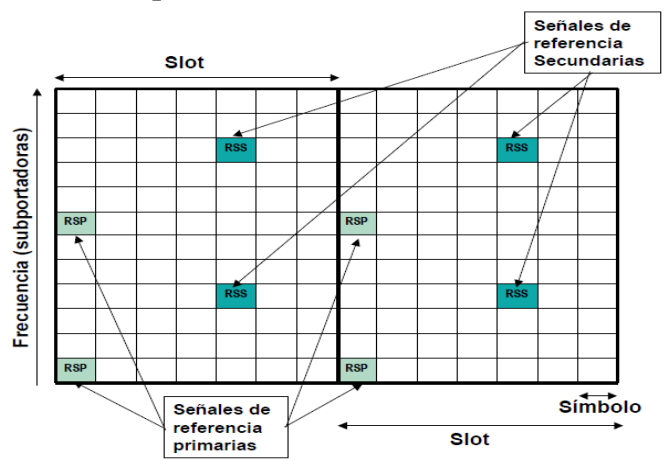

Fig. 11. Señales de referencia para una subtrama. (Agusti et al., 2010).

En la Fig. 11 se muestra un ejemplo de la ubicación de las señales RSP y RSS dentro de una subtrama genérica, compuesta por dos PRB, una antena única y 12 subportadoras (Dahlman, Parkvall, \& Skold, 2013).

Para el caso de multiplexado espacial con dos antenas se puede ver un ejemplo en la Fig. 12 con 12 subportadoras para una subtrama. Los recursos frecuencia-tiempo marcados con " $\mathrm{X}$ " indican que no se puede transmitir en ellos.
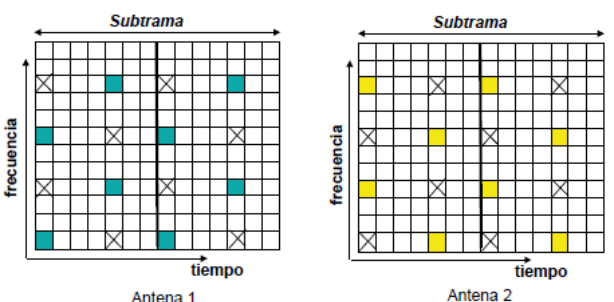

Fig. 12. Señales de referencia para una subtrama con 2 antenas. (Agusti et al., 2010). 
En la definición de los símbolos de referencia consideran los siguientes conceptos (Agusti et al., 2010):

1. La separación temporal de los símbolos de referencia depende de la máxima FD (Frequency Doppler) del canal que el sistema puede soportar, definida como la máxima velocidad con que el canal puede variar. El TC (tiempo de coherencia) del canal (es decir el tiempo en el que el canal no varía) es aproximadamente $\mathrm{TC}=1 / \mathrm{fd}$ y tomando en consideración el criterio de Nyquist, para poder reconstruir la respuesta implusional a partir de los símbolos de referencia será necesario ubicar al menos 2 símbolos por TC. TC $=1 \mathrm{~ms}$ y la separación mínima entre símbolos de referencia debe ser 0,5 ms.

2. En el dominio de la frecuencia, el sistema ubica un símbolo de referencia cada seis subportadoras. Esta separación está originada por el comportamiento del canal móvil. En entornos urbanos, la dispersión temporal del canal (medida en términos del Delay Spread o s S) es del orden de $1 \mu \mathrm{s}$. La banda de coherencia del canal es aproximadamente $\mathrm{BC}=1 / 10 \mathrm{~s} \mathrm{~S}$, es del orden de los $100 \mathrm{KHz}$. La separación de frecuencia entre símbolos de referencia es de 6 subportadoras $(90$ $\mathrm{KHz}$ ). En el caso de múltiples antenas en el transmisor, cada antena tiene su patrón de señales piloto, tal como se muestra en la Fig. 12.

\section{- $\quad \mathrm{SCH}$ (synchronization channel):}

La señal SCH se utiliza para facilitar los procesos de sincronización temporal a nivel de trama y subtrama del sistema (Agusti et al., 2010). Durante la búsqueda de la celda existe la necesidad de información específica sobre el UE, como el tiempo del frame, la frecuencia, la identificación de la celda, el ancho de banda de transmisión, la configuración de la antena y el prefijo cíclico. Además de las señales de referencia, las señales de sincronización son requeridas durante la búsqueda de la celda. En E-UTRA (Evolved UMTS Terrestrial Radio Access) la sincronización y el identificador de grupo de celdas se obtienen de los canales SCH (Somasegaran, 2007).

La estructura predefinida de estos canales son 72 subportadoras centradas alrededor de una subportadora en el frecuencia central $\mathrm{f}=0$ con $\operatorname{los}$ slots predefinidos para esa trama. $\mathrm{PSCH}$ y $\mathrm{SSCH}$ son utilizados en el penúltimo y antepenúltimo símbolos de OFDM respectivamente. La búsqueda de celda siembre se lleva a cabo usando las 72 subportadoras centrales independiente del ancho de banda de transmisión. Estas señales se dividen en (Agusti et al., 2010):

- P-SCH (Primary SCH) que permite la sincronización temporal a nivel de subtrama, mediante procedimientos de correlación entre la señal recibida y una secuencia de referencia almacenada en el receptor.

- $\mathrm{S}-\mathrm{SCH}$ (Secondary SCH) que posibilita la sincronización temporal a nivel de trama, utilizando la misma metodología de correlación temporal.

La señal P-SCH se genera a partir de una secuencia de Zadoff-Chude de longitud 63 a la que se le ha eliminado el elemento central (Soliman, 2009), (Kong, Lee, Han, Shin, \& You, 2008). Se transmite utilizando las 62 subportadoras centrales del ancho de banda utilizado por el sistema, dejando sin ocupar la subportadora central o portadora DC. Para separar esta señal de otras señales, se dejan 5 subportadoras vacías a su izquierda y derecha, que actúan como bandas de guarda.

La señal S-SCH siempre está asociada a una señal P-SCH. Cada secuencia secundaria de sincronización se obtiene como resultado de entrelazar, en el dominio de la frecuencia, una secuencias de máxima longitud de longitud 31 y su correspondiente versión desplazada cíclicamente como se observa en la Fig. 13. La modulación utilizada en cada subportadora es BPSK (Binary Phase Shift Keying).

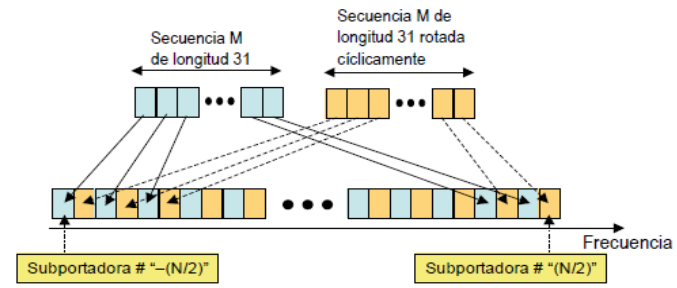

Fig. 13. Secuencia de sincronización secundaria. (Agusti et al., 2010).

Para facilitar la sincronización temporal a nivel de trama, la señal secundaria se transmite dos veces por trama. La secuencia de longitud 62 utilizada en cada símbolo no es exactamente la misma ya que difiere en la forma en que se genera. En la secuencia asignada al segundo símbolo, los papeles de las secuencias de máxima longitud de la Fig. 13 están intercambiados, es decir, los símbolos de la secuencia de máxima longitud original ocupan ahora las posiciones pares y los símbolos asociados a la secuencia rotada cíclicamente las posiciones impares de la secuencia de 62 símbolos. 
Al conectar el terminal móvil el primer procedimiento que éste realiza es el procedimiento de sincronización y adquisición inicial de parámetros, por lo cual las señales de sincronización primaria y secundaria se detectan en primer lugar, de modo que la identificación de celda indicada por éstas señales se utiliza para posteriormente identificar en qué recursos se van a situar las señales de referencia RSP y RSS (Agusti et al., 2010).

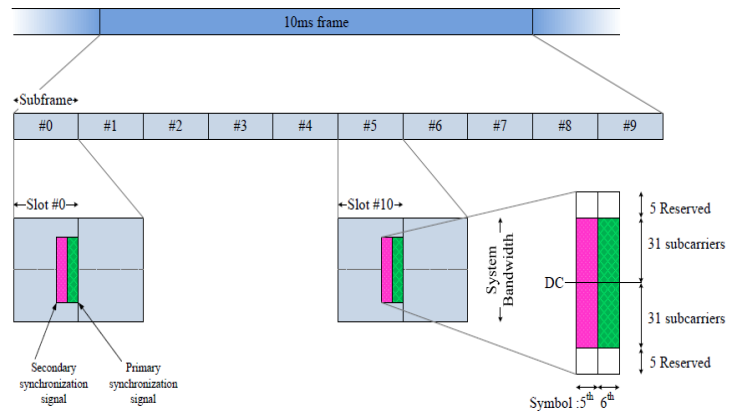

Fig. 14. Señales de sincronización en trama tipo 1.

(Agusti et al., 2010).

Hay que destacar que, al situar ambas señales de sincronización en el centro del radiocanal disponible, se facilita la sincronización de los terminales móviles a la red, independientemente de cual sea el número de subportadoras con las que dichos terminales móviles operen. El sistema permite un ancho de banda flexible entre 1.4 y 20 MHz (Agusti et al., 2010).

Si se utiliza una estructura de trama del tipo 1 o SF1 la señal P-SCH se transmite utilizando el último símbolo de del slot 0 y 10 (correspondientes a la primera y sexta subtrama), mientras que la señal S-SCH se transmite utilizando el penúltimo símbolo de los mismos slots, como se observa en la Fig. 14.

Si se utiliza una estructura de trama del tipo 2 o $\mathrm{SF} 2$, entonces la señal $\mathrm{P}-\mathrm{SCH}$ se transmite utilizando el campo DwPtS de la subtrama especial, mientras que la señal S-SCH se transmite utilizando el último símbolo OFDM de las subtramas numeradas como cero y cinco de dicha estructura de trama, como se observa en la Fig. 15.

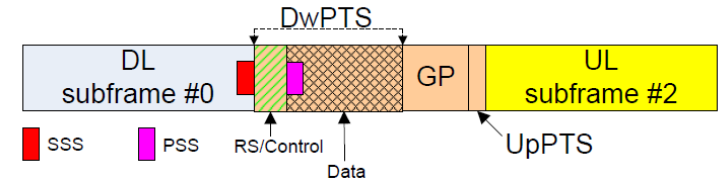

Fig. 15. Señales de sincronización en trama tipo 2. (Agusti et al., 2010).

\subsection{Canales Físicos en Downlink}

La señalización de capas altas y el tráfico de datos del usuario se organizan por medio de los canales adecuados para cada una. Los canales de DL se pueden observar en la Fig. 16 (Mannani, 2012). A continuación se describen los canales de enlace DL físico de LTE (Mannani, 2012):

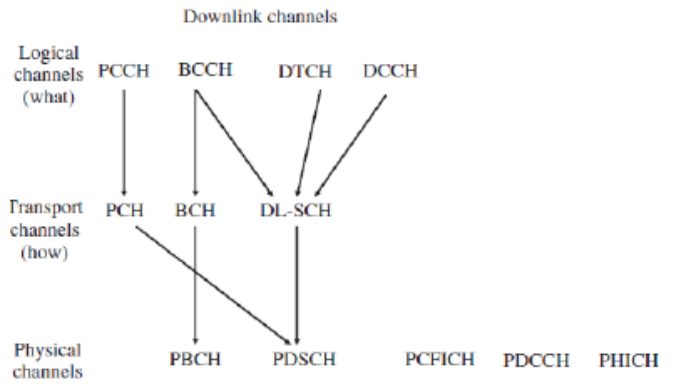

Fig. 16. Canales de DL de LTE (Mannani, 2012).

PBCH (Physical Broadcast Channel): El PBCH se utiliza para enviar parámetros de identificación del sistema y control de acceso de cada célula cada cuatro tramas $(40 \mathrm{~ms})$ utilizando QPSK. La estructura de $\mathrm{PBCH}$ es independiente del ancho de banda de la red.

PDSCH (Physical Downlink Shared Channel): El PDSCH se utiliza para transportar datos de usuario y está diseñado para altas velocidades de transferencia. Los bloques de recursos asociados con este canal se comparten entre los usuarios a través de OFDMA. Las diversas opciones de modulación incluyen QPSK, 16QAM y 64QAM. Para el PDSCH se utiliza la multiplexación espacial.

PCFICH (Physical Control Format Indicator Channel): se utiliza para informar al UE cuántos símbolos OFDM se utilizaran para la información de control en el PDCCH en una subtrama. El número de símbolos utilizados varía de 1 a 3 . El PCFICH utiliza modulación QPSK.

PDCCH (Physical Downlink Control Channel): se utiliza para informar al UE sobre la planificación de la asignación de recursos en el enlace UL y DL. Se asigna a los elementos de recursos hasta en los tres primeros símbolos OFDM en el primer slot de una subtrama y utiliza modulación QPSK. El valor de la PCFICH indica el número de símbolos utilizados para el PDCCH.

PMCH (Physical Multicast Channel): lleva información de difusión de multimedia con el uso de la modulación QPSK, 16 - QAM, o 64 - QAM. 
La información de multidifusión puede ser enviada a múltiples UE simultáneamente.

PHICH (Physical Hybrid Automatic Retransmission Request Indicator Channel) lleva el mensaje de Acknowledge (ACK)/Not Acknowledge (NACK) en el enlace DL en respuesta a las transmisiones de enlace UL con el fin de solicitar la retransmisión o confirmar la recepción de bloques de datos del UE. ACK y NACK se implementan en el mecanismo HARQ.

\section{ALGORITMOS DE PLANIFICACIÓN DE RECURSOS}

Dentro de las funciones RRM (Radio Resource Management) que tienen gran impacto sobre la eficiencia tenemos los planificadores de paquetes encargados de asignar en forma ordenada, los recursos de radio disponibles a los usuarios. Estos algoritmos cobran gran importancia si consideramos que LTE es una tecnología de transmisión de datos que utiliza canales compartidos. Por lo tanto, las estrategias utilizadas para la planificación de paquetes tienen un impacto directo sobre la QoS que el sistema está en capacidad de ofrecer al usuario (3GPP TS 36.211, 2009), (Agusti et al., 2010).

El proceso de planificación se encarga de asignar los RB a los usuarios conectados al sistema cada TTI. El planificador determina cuál bloque de recursos o subtrama dentro de la matriz de recursos va a ser asignado a cada usuario. Este esquema puede ser visualizado en la Fig. 17.

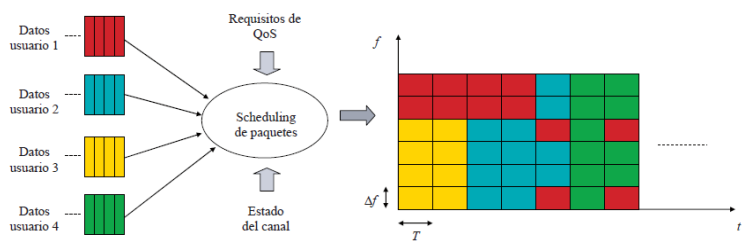

Fig. 17. Planificador de paquetes en OFDMA. (Agusti et al., 2010).

A cada usuario se le asigna una subtrama de la matriz de sub-portadoras de acuerdo con los parámetros de QoS, estado de canal y el algoritmo de planificación. Se pueden tener asignaciones de recursos en las que se da prioridad al usuario con mejor calidad de canal o se asigna proporcionalmente a todos los usuarios; esta asignación afecta directamente el rendimiento de la red LTE, puede dejar usuarios sin asignación o producir una velocidad muy baja de transmisión.
El proceso de selección de los parámetros MCS (Modulation And Coding Scheme) se conoce como LA (Link Adaptation). El LA junto con la programación de los recursos está destinada a maximizar la capacidad de la célula, mientras se asegura que se cumple con un mínimo de QoS y que hay recursos suficientes también para portadoras de best-effort sin requisitos estrictos de calidad de servicio (Holma \& Toskala, 2009). Los parámetros utilizados por LA pueden ser observados en la Fig. 18.

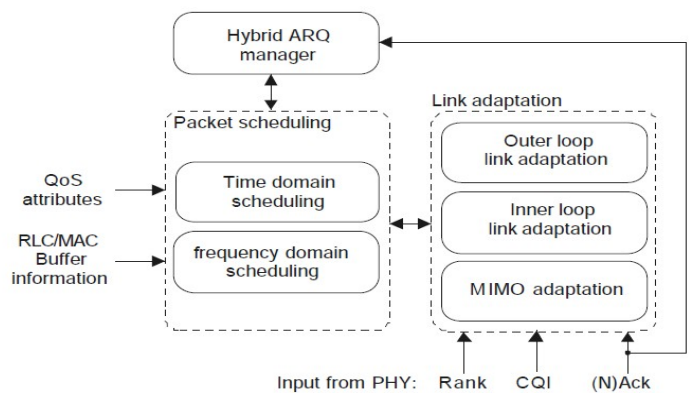

Fig. 18. Funcionalidades de la Capa 2 para planificación de paquetes dinámica, LA y HARQ.

(Holma \& Toskala, 2009).

LA ajusta la velocidad de datos con la ayuda del AMC (Adaptive Modulation and Coding), haciendo coincidir la modulación y el esquema de codificación de canal en los recursos asignados por el planificador (Mannani, 2012). El principio de funcionamiento puede verse en la Fig. 19 (Dahlman, Parkvall, Skold, \& Beming, 2010).

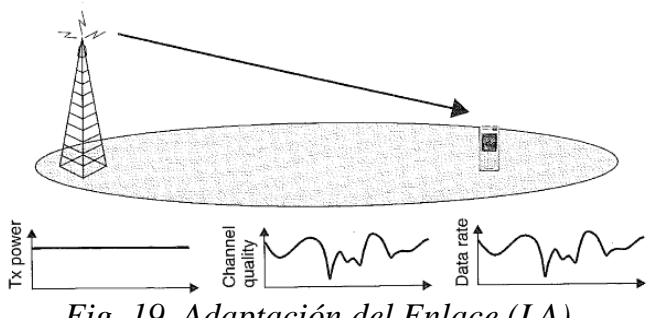

Fig. 19. Adaptación del Enlace (LA).

(Dahlman, Parkvall, \& Skold, 2013).

En principio, el eNodeB recibe periódicamente información CQI desde el terminal. Cuanto mayor sea el CQI, mejor es el canal. Por lo tanto basado en el CQI recibido desde el terminal, se puede realizar la AMC.

El terminal informa el CQI medido al eNodeB mediante la asignación de la medida SNR de acuerdo con la Fig. 20. En LTE, el mapa de SNR con respecto a CQI para un BLER de 0.1 se aproxima a través de una función lineal como se muestra en la Fig. 20 (Dikamba, 2011). 


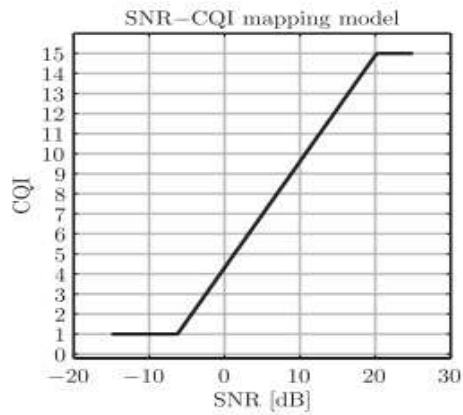

Fig. 20. Modelo de Mapa SNR vs CQI. (Dikamba, 2011).

La Tabla 2 contiene los valores del índice CQI, el esquema de modulación, la tasa de codificación y la eficiencia espectral para cada valor de CQI.

En LTE no existen canales de tráfico dedicados sino compartidos, los recursos son asignados a los usuarios únicamente cuando tienen datos para enviar o recibir. El planificador asigna el canal PDSCH (Physical Downlink Shared Channel), que transporta los datos de usuario en DL, cuando tienen información para recibir, o el canal PUSCH (Physical Uplink Shared Channel), que transporta los datos de usuario en UL, cuando los usuarios tienen información para enviar.

Tabla 2. Tabla de Valores de CQI (Dikamba, 2011)

\begin{tabular}{|c|c|c|c|}
\hline \hline $\begin{array}{c}\text { ÍNDICE } \\
\text { CQI }\end{array}$ & $\begin{array}{c}\text { MODULAC } \\
\text { IÓN }\end{array}$ & $\begin{array}{c}\text { TASA DE } \\
\text { CODIFICA } \\
\text { CIÓN }\end{array}$ & $\begin{array}{c}\text { EFICIENC } \\
\text { IA } \\
\text { [b/s/Hz] }\end{array}$ \\
\hline $\mathbf{0}$ & $\begin{array}{c}\text { Fuera de } \\
\text { Rango }\end{array}$ & $\begin{array}{c}\text { Fuera de } \\
\text { Rango }\end{array}$ & $\begin{array}{c}\text { Fuera de } \\
\text { Rango }\end{array}$ \\
\hline $\mathbf{1}$ & QPSK & $78 / 1024$ & 0.1523 \\
\hline $\mathbf{2}$ & QPSK & $120 / 1024$ & 0.2344 \\
\hline $\mathbf{3}$ & QPSK & $193 / 1024$ & 0.3770 \\
\hline $\mathbf{4}$ & QPSK & $308 / 1024$ & 0.6016 \\
\hline $\mathbf{5}$ & QPSK & $449 / 1024$ & 0.8770 \\
\hline $\mathbf{6}$ & QPSK & $602 / 1024$ & 1.1758 \\
\hline $\mathbf{7}$ & 16QAM & $378 / 1024$ & 1.4766 \\
\hline $\mathbf{8}$ & 16QAM & $490 / 1024$ & 1.9141 \\
\hline $\mathbf{9}$ & 16QAM & $616 / 1024$ & 2.4063 \\
\hline $\mathbf{1 0}$ & 64QAM & $466 / 1024$ & 2.7305 \\
\hline $\mathbf{1 1}$ & 64QAM & $567 / 1024$ & 3.3223 \\
\hline $\mathbf{1 2}$ & 64QAM & $666 / 1024$ & 3.9023 \\
\hline $\mathbf{1 3}$ & 64QAM & $772 / 1024$ & 4.4534 \\
\hline $\mathbf{1 4}$ & 64QAM & $873 / 1024$ & 5.1152 \\
\hline $\mathbf{1 5}$ & 64QAM & $948 / 1024$ & 5.5547 \\
\hline
\end{tabular}

En ambos casos la asignación se le comunica al usuario mediante el canal de control PDCCH (Physical Downlink Control Channel) para que puedan modular/demodular los canales de tráfico respectivos en el dominio tiempo-frecuencia que le fueron asignados. En el caso del UL, el móvil debe enviar su petición previamente al eNB para que el scheduler pueda asignarle los recursos, esto se lleva a cabo mediante el canal de control PUCCH (Physical Uplink Control Channel) a través de los Scheduling Request (Guinand Salas \& others, 2012).

Los procedimientos del Planificador de recursos en el UL para SC-FDMA son similares a los empleados en el DL para OFDMA. Sin embargo, debido a que estas funciones residen en el eNB, la asignación de los recursos en el UL es más compleja que en el enlace DL ya que requiere de mayor señalización del sistema.

Por ejemplo, el UE debe transmitir al eNB unas señales SRS (Sounding Reference Signals) para estimar la calidad del canal en cualquier PRB, además debe reportar el estado de su buffer enviando los BSR (Buffer Status Report) y debe informar su disponibilidad de potencia por medio de los mensajes PHR (Power Headroom Report) para que el planificador pueda establecer el esquema de modulación y codificación.

A diferencia del DL, en el UL las subportadoras asignadas a un usuario deben ser contiguas por basarse en SC-FDMA (Guinand Salas \& others, 2012). Este esquema se puede observar en la Fig. 21.

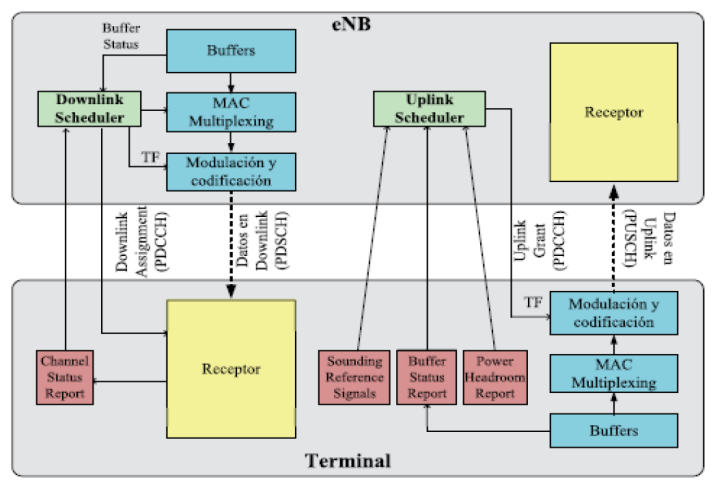

Fig. 21. Esquema para planificadores en LTE. (Guinand Salas \& others, 2012).

Para asignar los recursos de transmisión, se emplean diferentes algoritmos, como (García de la Torre \& Herradón Berzal, 2013):

\subsection{RR (Round Robin)}

La asignación de los recursos a los usuarios no tiene en cuenta las condiciones del canal. Se centra en el principio de fairness, ya que a todos los 
usuarios se les asigna la misma cantidad de recursos. La eficiencia es baja porque al no tener en cuenta las condiciones del canal, disminuye el throughput del sistema. El diagrama se puede observar en la Fig. 22.

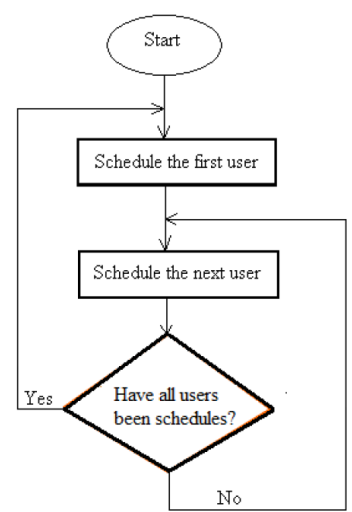

Fig. 22. Algoritmo Round Robin para planificación de recursos. (Dikamba, 2011)

\subsection{PF (Proportional-Fair)}

Este esquema tiene en cuenta las condiciones del canal. Su objetivo es conseguir un equilibrio entre el fairness y optimizar el throughput de cada usuario. El planificador asigna recursos al usuario que obtenga la mayor relación entre el throughput instantáneo y el throughput promedio acumulado.

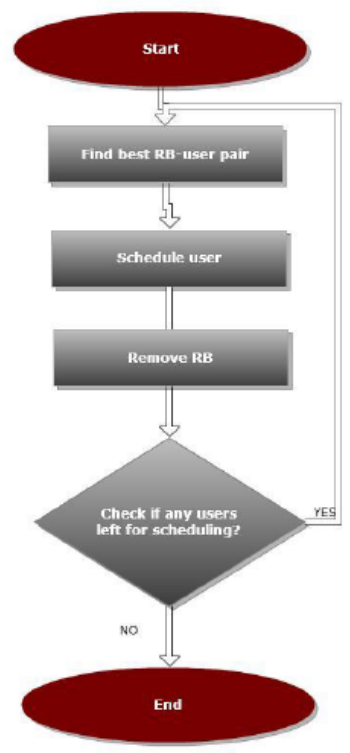

Fig. 23. Algoritmo PF para planificación de recursos (Mannani, 2012)

Hay diferentes implementaciones para el Proportional Fair. Uno de los mas utilizados se puede observar en la Fig. 23.

\subsection{Best CQI (Best Quality Indicators)}

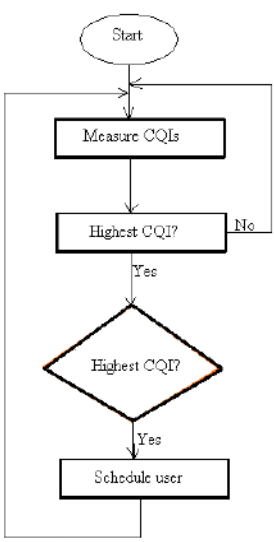

Fig. 24. Algoritmo Best CQI para planificación de recursos. (Mannani, 2012)

En este esquema el planificador asigna los recursos de radio a los usuarios con las mejores condiciones del canal. El eNodeB envía una señal de referencia (Downlink Pilot) a los UE. Estas señales son usadas para realizar las mediciones de CQI.

Entre más alto el valor de CQI, mejores condiciones tendrá el canal (Dikamba, 2011). Se consigue maximizar la capacidad de la celda, pero el fairness es bajo ya que los recursos solo estarán disponibles para los UE con las mejores condiciones del canal. En la Fig. 24 se observa el diagrama de bloques del algoritmo Best CQI.
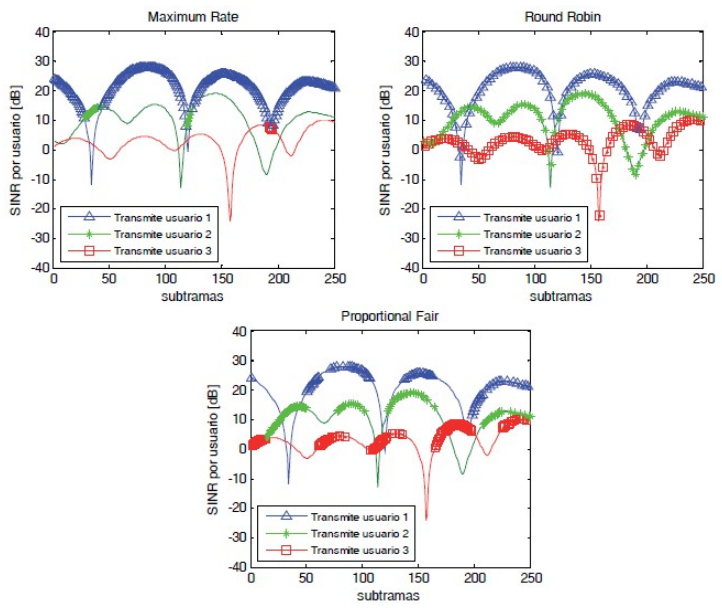

Fig. 25. Comparativa de Planificadores.

(García de la Torre \& Herradón Berzal, 2013)

En la Fig. 25, se aprecia el comportamiento de las técnicas mencionadas de planificación de recursos de los usuarios de LTE, en el momento en que cada uno de los tres usuarios transmiten de acuerdo al algoritmo elegido (García de la Torre \& Herradón Berzal, 2013). 
Basados en la Fig. 25 se puede apreciar que los algoritmos que toman en consideración el estado del canal como maximum Rate o Best CQI presentan niveles alto de throughput sacrificando el fairness pues a los 2 y 3 se les castiga por tener un SINR bajo.

Por otra parte algoritmos como RR asignan a todos los usuarios la misma cantidad de recursos, alcanzando niveles más altos de fairness pero sacrificando el throughput, algoritmos como PF busca obtener niveles de throughput y fairness equilibrados.

\section{CONCLUSIONES}

Mediante el análisis de la capa física, se logran identificar los diferentes elementos que componen los planificadores de recursos en LTE y WiMAX, como las diferentes tramas, sub-tramas y señales de sincronismo y de señalización que deben ser configuradas por los algoritmos de planificación de recursos del enlace descendente de LTE y WiMAX.

Los algoritmos que tienen en cuenta el estado del canal como Best CQI permiten lograr picos de throughput más elevados, pero al evaluar el Fairness se observa que su nivel es muy bajo. Esto se debe a que el algoritmo asigna una mayor cantidad de recursos a los usuarios que tienen un valor alto de CQI y a los demás los deja sin asignación, esto conlleva a que el algoritmo tenga un mayor rendimiento para los usuarios que se encuentran cerca al eNodeB, pero no se recomienda su uso para usuarios distantes al eNodeB.

Por otra parte, los algoritmos que asignan los recursos de radio de una manera más equitativa como RR y PF tienen índices de Fairness altos, pero hacen que el throughput para cada usuario sea bajo. Sin embargo, permiten que usuarios que se encuentren dentro de la zona de cobertura del eNodeB tengan recursos asignados.

Utilizando herramientas como el diseño CrossLayer y su inclusión en los algoritmos de planificación de recursos, se espera obtener algoritmos que permitan un mejor balance entre throughput y fairness para todos los usuarios conectados a la red, mejorando también temas como la calidad de la experiencia.

\section{REFERENCIAS}

3GPP TS 36.101. (2009). User Equiment (UE) radio transmission and reception.

3GPP TS 36.211. (2009). Evolved Universal Terrestrial Radio Access (E-UTRA): Physical Channels and Modulation.

Agusti, R., Bernardo, F., Casadevall, F., Ferrús, R., Pérez-Romero, J., \& Sallent, O. (2010). LTE: nuevas tendencias en comunicaciones móviles. Fundación Vodafone España.

Akan, A., \& Edemen, C. (2010). Path to 4G wireless networks (pp. 405-407). doi:10.1109/PIMRCW.2010.5670404

AlQahtani, S. A., \& Alhassany, M. (2013). Comparing different LTE scheduling schemes. In Wireless Communications and Mobile Computing Conference (IWCMC), 2013 9th International (pp. 264-269).

Castellanos, G. D., \& Khan, J. Y. (2010a). Performance of WiMAX packet schedulers for multi-class traffic. Communications (LATINCOM), 2010 IEEE Latin-American Conference on, 1-6.

Castellanos, G. D., \& Khan, J. Y. (2010b). Performance of WiMAX packet schedulers for multi-class traffic. In 2010 IEEE LatinAmerican Conference on Communications (LATINCOM) (pp. 1-6). doi:10.1109/LATINCOM.2010.5640994

Chakchai So-In, Jain, R., \& Tamimi, A.-K. (2009). Scheduling in IEEE 802.16e mobile WiMAX networks: key issues and a survey. Selected Areas in Communications, IEEE Journal on, 27(2), 156-171.

Cox, C. (2012). An introduction to LTE: LTE, LTEadvanced, $S A E$ and $4 G$ mobile communications. John Wiley \& Sons.

Dahlman, E., Parkvall, S., \& Skold, J. (2013). 4G: LTE/LTE-advanced for mobile broadband. Academic Press.

Dahlman, E., Parkvall, S., Skold, J., \& Beming, P. (2010). $3 G$ evolution: HSPA and LTE for mobile broadband. Academic press.

Dikamba, T. (2011). Downlink Scheduling in 3GPP Long Term Evolution (LTE). Delft University of Technology.

Fang, Y., \& McDonald, A. B. (2004). Dynamic codeword routing (DCR): a cross-layer approach for performance enhancement of general multi-hop wireless routing. In Sensor and Ad Hoc Communications and Networks, 2004. IEEE SECON 2004. 2004 First Annual IEEE Communications Society Conference on (pp. 255-263). 
Furht, B., \& Ahson, S. A. (2009). Long Term Evolution: $3 G P P$ LTE radio and cellular technology. Crc Press.

G. Israel. (2013). Gestión de Recursos Radio en Redes Móviles Celulares Basadas en Tecnología OFDMA para la Provisión de QoS y Control de la Interferencia. Universidad de Zaragoza.

García de la Torre, H., \& Herradón Berzal, G. (2013). Estudio y desarrollo sobre sistemas de simulación para comunicaciones móviles, LTE.

Guinand Salas, C. E., \& others. (2012). Planificación de una red LTE con la herramienta ATOLL y análisis del impacto de las estrategias de Packet Scheduling.

Holma, H., \& Toskala, A. (2009). LTE for UMTSOFDMA and SC-FDMA based radio access. John Wiley \& Sons.

Ikuno, J. C., Wrulich, M., \& Rupp, M. (2010). System Level Simulation of LTE Networks. In Vehicular Technology Conference (VTC 2010Spring), 2010 IEEE 71st (pp. 1-5). doi:10.1109/VETECS.2010.5494007

Kong, K.-S., Lee, W., Han, Y.-H., Shin, M.-K., \& You, H. (2008). Mobility management for all-IP mobile networks: mobile IPv6 vs. proxy mobile IPv6. Wireless Communications, IEEE, 15(2), 36-45. doi:10.1109/MWC.2008.4492976

Mannani, D. (2012). Modeling and Simulation of Scheduling Algorithms in LTE Networks. Warsaw University of Technology.
Melodia, T., Vuran, M., \& Pompili, D. (2006). The State of the Art in Cross-Layer Design for Wireless Sensor Networks. In M. Cesana \& L. Fratta (Eds.), Wireless Systems and Network Architectures in Next Generation Internet (Vol. 3883, pp. 78-92). Springer Berlin Heidelberg. http://dx.doi.org/10.1007/11750673_7

Motorola. (White Paper). Long Term Evolution (LTE): Overview of LTE Air-Interface.

Nuaymi, L. (2007). WiMAX: technology for broadband wireless access. John Wiley \& Sons.

Soliman, H. (2009). Mobile IPv6 support for dual stack hosts and routers.

Somasegaran, L. (2007). Channel estimation and prediction in UMTS LTE. Aalborg University, Institute of Electronic Systems, Signal and Information Processing for Communications.

Taha, A.-E. M., Ali, N. A., \& Hassanein, H. S. (2011). LTE, LTE-Advanced and WiMAX: Towards IMT-Advanced Networks. John Wiley $\&$ Sons.

Ukil, A. (2009). Cross-layer optimization in QoS aware next generation wireless networks. In Information, Communications and Signal Processing, 2009. ICICS 2009. 7th International Conference on (pp. 1-5). doi:10.1109/ICICS.2009.5397495

Van Hoesel, L., Nieberg, T., Wu, J., \& Havinga, P. J. M. (2004). Prolonging the lifetime of wireless sensor networks by cross-layer interaction. Wireless Communications, IEEE, 11(6), 78-86. doi:10.1109/MWC.2004.1368900

Vuran, M. C., Gungor, V. C., \& Akan, O. B. (2005). On the interdependency of congestion and contention in wireless sensor networks ( $\mathrm{pp}$. 136-147). 